\title{
Entangling COVID-19 associated thrombosis into a secondary antiphospholipid antibody syndrome: Diagnostic and therapeutic perspectives (Review)
}

\author{
EUGENIO CAVALLI ${ }^{1}$, ALESSIA BRAMANTI $^{2}$, ROSELLA CIURLEO ${ }^{2}$, \\ ANDREY I. TCHORBANOV ${ }^{3,4}$, ANTONIO GIORDANO ${ }^{5,6}$, PAOLO FAGONE ${ }^{1}$, CRISTINA BELIZNA ${ }^{7,8}$, \\ PLACIDO BRAMANTI $^{2}$, YEHUDA SHOENFELD ${ }^{9,10}$ and FERDINANDO NICOLETTI ${ }^{1}$
}

\author{
${ }^{1}$ Department of Biomedical and Biotechnological Sciences, University of Catania, I-95123 Catania; \\ ${ }^{2}$ IRCCS Centro Neurolesi ‘Bonino-Pulejo’, I-98124 Messina, Italy; ${ }^{3}$ Laboratory of Experimental Immunology, \\ Institute of Microbiology, Bulgarian Academy of Sciences; ${ }^{4}$ National Institute of Immunology, 1113 Sofia, Bulgaria; \\ ${ }^{5}$ Sbarro Institute for Cancer Research and Molecular Medicine, Center for Biotechnology, College of Science and Technology, \\ Temple University, Philadelphia, PA 19122, USA; ${ }^{6}$ Department of Medical Biotechnologies, University of Siena, \\ I-53100 Siena, Italy; ${ }^{7}$ Vascular and Coagulation Department, University Hospital Angers, 49000 Angers; \\ ${ }^{8}$ MITOVASC Institute and CARFI Facility, University of Angers, UMR CNRS 6015, INSERM U1083, 49045 Angers, \\ France; ${ }^{9}$ Zabludowicz Center for Autoimmune Diseases, Sheba Medical Center, Affiliated to Tel-Aviv University, \\ Ramat Gan 5265601, Israel; ${ }^{10}$ I.M. Sechenov First Moscow State Medical University of the Ministry \\ of Health of the Russian Federation (Sechenov University), 119991 Moscow, Russia
}

Received June 17, 2020; Accepted June 25, 2020

DOI: $10.3892 / \mathrm{ijmm} .2020 .4659$

\begin{abstract}
The severe acute respiratory syndrome coronavirus 2 (SARS-CoV-2) is a novel $\beta$ coronavirus that is the etiological agent of the pandemic coronavirus disease 2019 (COVID-19) that at the time of writing (June 16, 2020) has infected almost 6 million people with some 450,000 deaths. These numbers are still rising daily. Most (some $80 \%$ ) cases of COVID-19 infection are asymptomatic, a substantial number of cases (15\%) require hospitalization and an additional fraction of patients $(5 \%)$ need recovery in intensive care units. Mortality for COVID-19 infection appears to occur globally between 0.1 and $0.5 \%$ of infected patients although the frequency of lethality is significantly augmented in the elderly and in patients with other comorbidities. The development of acute respiratory distress syndrome and episodes of thromboembolism that may lead to disseminated intravascular coagulation (DIC) represent the primary causes of lethality during COVID-19 infection. Increasing evidence suggests that thrombotic diathesis is due
\end{abstract}

Correspondence to: Professor Ferdinando Nicoletti, Department of Biomedical and Biotechnological Sciences, University of Catania, Via S. Sofia 89, I-95123 Catania, Italy

E-mail: ferdinic@unict.it

Key words: acute respiratory distress syndrome, anti-cardiolipin autoantibodies, anti- $\beta 2$ glycoprotein 1 autoantibodies, anti-phospholipid autoantibodies, anti-phospholipid syndrome, coagulopathy, COVID-19, disseminated intravascular coagulation, severe acute respiratory syndrome coronavirus 2 , pneumonia, thromboembolism, stroke to multiple derangements of the coagulation system including marked elevation of D-dimer that correlate negatively with survival. We propose here that the thromboembolic events and eventually the development of DIC provoked by SARS-CoV-2 infection may represent a secondary anti-phospholipid antibody syndrome (APS). We will apply both Baconian inductivism and Cartesian deductivism to prove that secondary APS is likely responsible for coagulopathy during the course of COVID-19 infection. Diagnostic and therapeutic implications of this are also discussed.

\section{Contents}

1. Introduction

2. The variable clinical courses of COVID-19 infection

3. Aim of the review

4. Coagulopathy, thromboembolic events and DIC during COVID-19 infection

5. Can the thromboembolic diathesis during some cases of COVID-19 represents secondary form of anti-phospholipid antibody syndrome? Combining Cartesio deductivism and Baconian inductivism to prove the hypothesis

6. Future therapeutic directions

\section{Introduction}

The severe acute respiratory syndrome coronavirus 2 (SARS-CoV-2) is the etiological agent of the coronavirus disease 2019 (COVID-19) that was declared as a global 
pandemic on March 11, 2020. At the time of writing (June 16, $2020)$ it has infected almost 6 million people with some 450,000 deaths, with numbers rising daily.

Coronaviruses are single-stranded RNA viruses that can infect several host species (1) and can be subdivided into $\alpha$, $\beta, \gamma$, and $\delta$ genera, and with SARS-CoV, Middle East respiratory syndrome coronavirus (MERS-CoV) and SARS-CoV-2 belonging to $\beta$ coronaviruses (1).

Similar to SARS-CoV, SARS-CoV-2 mainly transmits through respiratory droplets and direct contact $(2,3)$. However, SARS-CoV-2 seems more infective but less virulent than SARS-CoV $(4,5)$ as it is also consistent with its lower rate of lethality, that seems to range between 0.3 and $0.9 \%$ of infected patients (1-4). Like SARS-CoV and other coronaviruses, SARS-CoV-2 enters into the host cells through binding with the receptor via the S-spike on the surface of the virus (4). In addition, SARS-CoV-2 is uniquely endowed with a furin cleavage site ('RPPA' sequence) at the S1/S2 site that is likely responsible of its strong pathogenicity (1). In a manner similar to SARS-CoV, SARS-CoV-2 employs the angiotensin-converting enzyme 2 (ACE2) as its receptor but with ten times higher affinity than the former (6-8). ACE2 is abundantly expressed in different cells of the lung, heart, ileum, kidney, bladder and brain and this may explain the variable clinical symptomatology induced by SARS-CoV-2 infection (6).

\section{The variable clinical courses of COVID-19 infection}

Clinical features. COVID-19 manifests with a wide clinical spectrum ranging from asymptomatic patients to septic shock and multi-organ dysfunction. Interestingly, it has been reported that smell and taste dysfunction are associated with COVID-19 (7).

COVID-19 infection is classified into mild, moderate, severe, and critical depending on the symptoms that are shown in Table I. The asymptomatic or mild course is seen in some $80 \%$ of the patients, another $15 \%$ experience serious course requiring hospitalization and $5 \%$ have a critical illness. The symptoms appear after an incubation period of approximately 1 week (8). The period from the onset of COVID-19 symptoms to death varies from 1 to 6 weeks with a median of 14 days (9). This time-frame depends on the age of the patient and the presence of other comorbidities being shorter among patients $>70$-years old (9). It has been reported that for critical Chinese patients the case fatality rate is $49 \%$ (10), that is increased by preexisting comorbidities such as diabetes $(7.3 \%)$, respiratory disease $(6.5 \%)$, cardiovascular disease $(10.5 \%)$, hypertension (6\%), and oncological complications (5.6\%) (11). The lack of comorbidities markedly lowers the case fatality rate to $0.9 \%$ (11). The clinical manifestations of COVID-19 infection in the different clinical course of the disease are synoptically summarized in Table I.

\section{Aim of the review}

This review will focus on the occurrence of mild to severe derangement of the coagulation system that may range from inapparent thrombosis to arterial and venous thrombosis in multiple sites and organs to potentially lethal disseminated intravascular coagulation (DIC).
On the basis of empirical observations and emerging laboratoristic findings, we will elaborate the hypothesis that several cases of thrombotic events during COVID-19 infection represent the clinical epiphenomenon of a viral-induced secondary anti-phospholipid antibody syndrome (APS) that, in the most severe cases, may develop as catastrophic anti-phospholipid antibody syndrome (CAPS). Diagnostic and therapeutic consequences of this are discussed.

\section{Coagulopathy, thromboembolic events and DIC during COVID-19 infection}

Clinical evidence and emerging data from pathological examinations indicate that a thrombotic diathesis, potentially leading to venous thromboembolism (VTE), and to DIC in some of the most severe cases, may occur in a substantial proportion of patients with COVID-19 infection, also in a manner independent of long-term bed rest and eventual hormonal treatment. We will discuss, in this chapter, laboratoristic analyses, clinical evidences and interventional studies with anticoagulant therapies that lend support to the concept that the coagulation system is severely deranged during COVID-19 infection and may play a key role in determining the severity of the disease and its rate of lethality.

Laboratoristic analyses. In an initial and important study, Tang et al (12) retrospectively analyzed conventional coagulation results and outcomes of 183 consecutive patients with confirmed COVID-19 infection. The study demonstrated that, when evaluated at baseline levels on hospital admission, the patients that died during the course of the infection by COVID-19 had higher levels of D-dimer and fibrin degradation products (FDP), along with longer prothrombin and activated partial thromboplastin times than survivors. In addition, $71.4 \%$ of non-survivors and $0.6 \%$ survivors met the criteria of DIC.

This study attracted much attention on the occurrence and pathogenically significant role of abnormal coagulation results during severe COVID-19 infection (12).

Lending support to the pathogenic implication of the abnormal coagulation pathways during COVID-19 infection was a meta-analysis carried out by Li et al (13) on 10 studies entailing a total of 1,995 cases that reported a significant increase of D-dimer in a substantial number of patients. Along this line of research, Zou et al (14) evaluated retrospectively the abnormalities of the coagulation system and correlated them with the disease status. The patients were divided into two groups with mild and severe disease. More males (76.9 vs. $49.8 \%$ ) and older patients (median age 65 vs. 50) and higher frequency of other comorbidities were observed in patients with severe disease. Altogether, 209 abnormalities $(69.0 \%)$ of coagulation indexes were observed in the cohort of 303 patients and were more frequent in patients affected by severe disease (100 vs. 66.1\%). The international normalized ratio, the prothrombin time, the activated partial thromboplastin time, the fibrinogen, the FDP, and the D-dimer were all significantly augmented in the patients with severe diseases as compared to those with mild disease. This study further and clearly supports the concept that coagulation dysfunction, in particular fibrinogen and D-dimer elevation, is common in 
Table I. Clinical manifestation of SARS-CoV-2 infection.

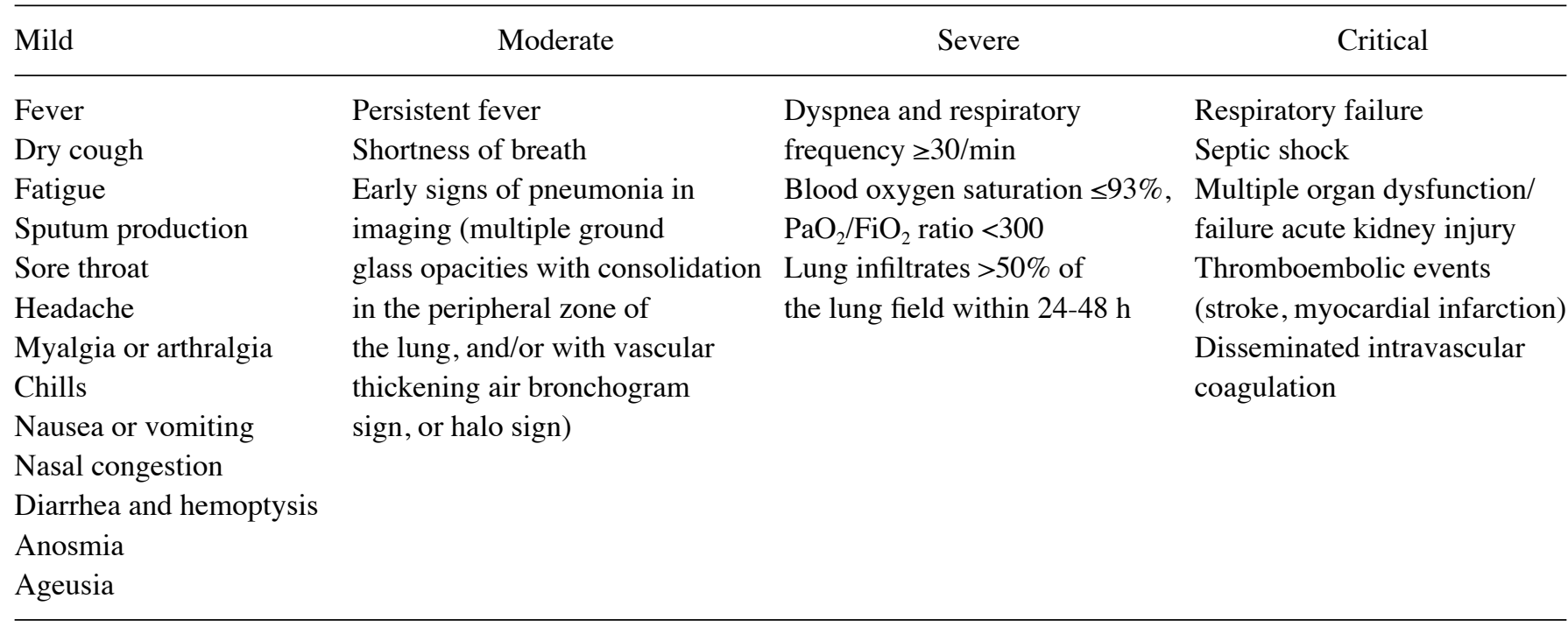

SARS-CoV-2, severe acute respiratory syndrome coronavirus 2 .

patients with COVID-19, and the degree of elevation is related to the severity of the disease. The reduction of both fibrinogen and activated partial thromboplastin time are associated with recovery (14).

Clinical evidence. Propelled from these laboratoristic observations, several clinical studies investigated the role of the abnormalities of coagulation system during COVID-19 infection. An Italian study evaluated symptomatic patients with laboratory-proven COVID-19 (15). A total of 388 patients were recruited. In spite of the thromboprophylaxis administered to all patients, thromboembolic events occurred in 28 $(21 \%)$ of them. Forty-four patients underwent VTE imaging tests, that were confirmed in $16(36 \%)$. Pulmonary embolism was confirmed in 10 out of 30 patients (33 and $7.7 \%$ of total). The rate of ischemic stroke and acute coronary syndrome /myocardial infarction was 2.5 and $1.1 \%$, respectively. Overt DIC was present in $8(2.2 \%)$ patients. This study demonstrates that venous and arterial thromboembolic events is frequent during COVID-19 infection and independent of thromboprophylaxis and that $50 \%$ of events are diagnosed within $24 \mathrm{~h}$ of hospital admission. In addition out of the $11 \%$ of total patients undergoing VTE imaging tests, 16 were positive (36\% of tests), suggesting an underestimation of thromboembolic complications (15).

Therapeutic intervention with anticoagulant therapies. That thromboembolism is involved in the clinical course of COVID-19 infection concurs with the reduction of mortality rate observed in one study that treated COVID-19 infected patients with anticoagulant treatment (16). Another retrospective study was conducted on 449 patients with severe COVID-19 and 99 of them were on heparin for 7 days or longer (17). The 28-day mortality rate was positively associated to D-dimer, prothrombin time, and age and negatively with platelet count. Interestingly, the 28-day mortality of heparin users was lower than non-users in patients stratified by the sepsis-induced coagulopathy (SIC) score or D-dimer result with SIC score $\geq 4$, or D-dimer $>6$-fold of the upper limit of normal. These data represent a valuable proof of concept for biomarker driven approach to heparin use in patients infected with COVID-19 (17).

Evidence is also emerging that ethnicity has major effects on thrombotic risk, with a 3-4-fold lower risk in Chinese compared to Caucasians and a significantly higher risk in African-Americans. When studying coagulopathy in Caucasian patients infected with COVID-19, Fogarty et al (18) demonstrated that, when treated with low molecular weight heparin, these patients rarely develop overt DIC, which is eventually limited to the late stage of the disease. The authors also propose that the diffuse bilateral pulmonary inflammation observed in COVID-19 is associated with a novel pulmonary-specific vasculopathy, named intravascular coagulopathy (PIC). In agreement with the well-established thrombotic diathesis of COVID-19 patients, it seems possible that PIC may contribute to the unexplained emerging differences that highlight racial susceptibility to COVID-19 mortality.

Taken as a whole, these laboratoristic and clinical observations and both prospective and retrospective outcomes from interventional studies employing anticoagulant therapies indicate an important and probably underestimated role of thromboembolic complications during COVID-19 and warns on the urgent necessity of proper diagnostic and therapeutic monitoring of the coagulation system during the infection. Particularly so in those COVID-19 infected patients with preexisting thrombotic disease, or those who need prevention or care for their thrombotic disease during the COVID-19 pandemic (16).

Nonetheless, a recent study demonstrated occurrence of heparin resistance in some patients with COVID-19 infection as defined by the need of dose unfractionated heparin of more than 35,000 IU/day to achieve the target aPTT ratio or the impossibility of doing so (19). Heparin resistance in these patients was associated to highly increased levels of Factor 
VIII level, fibrinogen and d-dimer while almost all of the antithrombin levels were in the normal range (19).

The emerging role of cytokines complement as effector mechanisms of inflammation and thrombosis during COVID-19. Increasing evidence indicates that upregulated release of proinflammatory cytokines of the innate immune system secreted either in the vicinity of organ targeted from the virus (e.g., the alveolar cells of the lung) or in the peripheral circulation in response to infection by SARS-CoV-2 may represent the culprit of the immunoinflammatory process (20). Evidence for the occurrence of a cytokine storm during the occurrence of SARS-CoV-2 infection and its pathogenic role in determining immunoinflammatory pneumonia and thrombosis have been repeatedly described with independent confirmation of augmented circulating levels of these proinflammatory cytokines (20). These observations led to the adoption of the anti-IL-6 receptor monoclonal antibody tocilizumab for the treatment of pneumonia associated to cytokine storm. It has also been demonstrated that the local immunoinflammatory response triggered from these cytokines may lead to complement activation that may amplify the circuit of immunoinflammation and thrombosis (21). In fact, skin and lung tissues from 5 patients with severe COVID-19 associated with respiratory failure $(n=5)$ and purpuric skin rash $(n=3)$ had marked deposition of different terminal complement components in the microvasculature, and co-localization of COVID-19 spike glycoproteins with C4d and C5b-9 in the interalveolar septa and the cutaneous microvasculature of 2 cases examined (22). In a similar manner, the purpuric skin lesions exhibited a pauci-inflammatory thrombogenic vasculopathy, with deposition of C5b-9 and C4d in both grossly involved and normally-appearing skin. These observations suggest that at least certain cases of severe COVID-19 may be secondary to activation of the complement that leads to catastrophic microvascular injury and an associated procoagulant state. That complement may represent an important therapeutic target for the treatment of severe cases of COVID-19 has been suggested (22-24). A case of a patient with severe ARDS due to COVID-19 pneumonia who was successfully treated with the complement C3 inhibitor AMY-101 has been reported (25).

\section{Can the thromboembolic diathesis during some cases of COVID-19 represents secondary form of anti-phospholipid antibody syndrome? Combining Cartesio deductivism and Baconian inductivism to prove the hypothesis}

Background. The APS is characterized by the occurrence of multiple episodes of venous and arterial thromboses and recurrent fetal losses, frequently accompanied by a moderate thrombocytopenia, in the presence of antiphospholipid (auto) antibodies (aPL Abs) that are directed against cardiolipin $(\mathrm{aCL})$ or $\beta 2$ glycoprotein1 ( $\beta 2-\mathrm{GP} 1)$ (26). Activation of the complement is also required for the full clinical manifestation of the APS.

APS can occur idiopathically or it can be associated with other autoimmune diseases such as systemic lupus erythematosus (26). Catastrophic antiphospholipid syndrome (CAPS) is a severe manifestation of APS (26). Although affecting only
$1 \%$ of patients with APS, the condition is frequently fatal if not recognized and treated early.

Secondary cases of APS due to viral infections have been reported. Secondary cases of APS due to infectious agents potentially evolving into CAPS have been reported and include infections from hepatitis C virus, herpes zoster, as well as bacteria, fungi and parasites and acute $\mathrm{Q}$ fever (27).

The induction of molecular mimicry that leads to production of anti- $\beta 2$-GPI autoantibodies has been proposed as putative cause of secondary APS and CAPS $(28,29)$.

The immunopathogenetic mechanisms that are subsequently activated entail a network of multiple proinflammatory factors including the Toll-like receptor 4 (TLR-4), which triggers a cytokine storm, followed by endothelial alterations that induce a procoagulant state (30).

Diagnosis and classification criteria of APS. According to the original criteria formulated in Sapporo in 1988 and revised in Sidney in 2004, APS can be diagnosed in the presence of least one clinical (vascular thrombosis or pregnancy morbidity) and one laboratory including aCL, or anti- $\beta 2$-GPI autoantibodies with lupus anticoagulant (LA) (31). LA is a somehow enigmatic laboratoristic phenomenon observed in patients with APS and represents a paradox that is still unsolved. LA causes a phospholipid-dependent prolongation of the clotting time but is associated with an increased risk of thrombosis and pregnancy morbidity (32). Recently, Pengo et al (32) have demonstrated that LA positivity may identify two different groups of patients with or without anti- $\beta 2-\mathrm{GPI}\left(\mathrm{LA}^{+} / \mathrm{anti}-\beta 2-\mathrm{GPI}^{+}\right.$and $\mathrm{LA}^{+} /$anti- $\beta 2$-GPI ${ }^{-}$). The $\mathrm{LA}^{+} / \mathrm{anti}-\beta 2-\mathrm{GPI}-$ group of patients had anti-phosphatidylserine/prothrombin autoantibodies and consisted of significantly older patients, with a lower rate of previous thromboembolic events and a weaker LA activity.

Epidemiology. The incidence of the APS is reported as approximately 5 new cases per 100,000 persons per year and the prevalence approximately 40-50 cases per 100,000 persons. The aPL Abs are positive in approximately $13 \%$ of patients with stroke, $11 \%$ with myocardial infarction, $9.5 \%$ of patients with deep vein thrombosis and $6 \%$ of patients with pregnancy morbidity $(33,34)$. As it occurs for other autoimmune diseases, the presence of autoantibodies directed against CL and/or $\beta 2$-GPI has been observed in a percentage of healthy individuals without clinical symptoms of APS ranging from 4.5 to $5.5 \%$ (35). In addition, the prevalence of anti-CL autoantibodies varies with age, having been reported of $2 \%$ in young healthy individuals as compared to $12 \%$ of the elderly (mean age 70) healthy individuals (36). In a recent study conducted in 956 elderly individuals (mean age of 81.1 years; $72 \%$ women) positivity for a PL Abs including aCL, anti- $\beta 2$-GPI and antiphosphatidyl-serine autoantibodies was found in 197 (20.6\%) of them (37).

What converts aPL $A b(s)$ positive healthy individual into APS patients? In agreement to the second hit hypothesis, it is thought that inflammatory events, the use of tobacco, alcohol or obesity and the associated metabolic syndrome may trigger the full clinical development of APS. These risk factors may be observed in up to $50 \%$ of patients with APS. 
Table II. Similarities between COVID-19 and thrombosis/APS.

Antiphospholipid syndrome

(revised Sydney classification criteria)
COVID-19

Significantly higher D-dimer and FDP levels, longer prothrombin time and activated partial thromboplastin time in non-survivor as compared to survivors on admission. $71.4 \%$ of non-survivors and $0.6 \%$ survivors met the criteria of disseminated intravascular coagulation (12)

Abnormal coagulation parameters in $69.0 \%$ (out of 303) cases: FIB, D-dimer, prolonged PT, altered APTT, elevated FDP. Median INR, PT, APTT, FIB, FDP, and D-dimer significantly higher in the COVID-19 severe group compared to the mild group (14) Thromboembolic events among COVID-19 patients occurred at a cumulative rate of $21 \%$ (15)

Pulmonary involvement Large-vessel stroke in five patients younger than 50 years of age (57) Pulmonary intravascular coagulopathy (18) Deposits of complement components C5b-9, $\mathrm{C} 4 \mathrm{~d}$, and MASP2, in the microvasculature of lung and skin (22)

Pregnancy morbidity

Disregulated production of cytokine in APS cytokine storm in CAPS Anticardiolipin IgG and/or IgM Anti- $\beta 2-G P 1$ IgG and/or IgM Lupus anticoagulant
Cytokine storm

Anticardiolipin IgA antibodies and anti- $\beta 2-\mathrm{GP} 1$

$\operatorname{Ig} \mathrm{A}$ and $\mathrm{IgG}$ antibodies in three COVID-19 patients (45)

Lupus anticoagulant $(50,51)$

COVID-19, coronavirus disease 2019; APS, anti-phospholipid antibody syndrome; APTT, activated partial thromboplastin time; FB, fibrinogen; PT, prothrombin time; FDP, fibrinogen degradation products; MASP2, mannose binding lectin (MBL)-associated serine protease; CAPS, catastrophic anti-phospholipid antibody syndrome.

Findings from an epidemiological study showed that the risk of myocardial infarction or stroke in young women with LA is increased in those who smoke or take oral oestrogenic therapy.

As discussed above for development of secondary CAPS, it is proposed that after activation of endothelial cells, monocytes, and platelets by aPL Abs, a procoagulant state is induced, which is mainly mediated by the increased synthesis of tissue factor and thromboxane A2. Activation of the complement cascade might close the loop and provoke thrombosis, often in the presence of a second hit (38).

Clinical manifestations of APS. APS is characterized by arterial, venous, or small vessel thrombosis and/or recurrent early pregnancy loss, fetal loss, or pregnancy morbidity in the presence of aPL Abs that include the lupus anticoagulant, or moderate-high titer of aCL or anti- $\beta 2-$ GPI autoantibodies (26). CAPS is characterized by thrombosis in multiple organs and a cytokine storm developing over a short period, with histopathologic evidence of multiple microthromboses, and laboratory confirmation of high aPL Abs titers and it is characterized as widespread acute thrombotic microangiopathy $(26,39)$.
Standard of care (SOC) treatment for APS. The only approved SOC treatment for APS relies on the use of indefinite anticoagulation with a vitamin $\mathrm{K}$ antagonist as the standard care (26).

In addition, several anecdotic reports have proposed beneficial effects of immunomodulatory agents including corticosteroids, rituximab, IvIg, D3 vitamin, plasmapheresis and chloroquine, anti-complement antibody eculizumab and the mTOR inhibitor rapamycin $(26,40,41)$.

Can thrombosis during COVID-19 and APS be the same face of two different coins? Exercizing with Cartesio and the restricted but initiating power of the deductivism. From what we have stated above there are several similar clinical characteristics that associate COVID-19 and APS and that culminate with thrombosis and which may represent the ultimate clinical outcome of eventually overlapping immunopathogenic pathways.

The 3 main culprits responsible for activating immunoinflammatory responses and thrombosis appear to be the same in both diseases, namely upregulated cytokine secretion from cells of the innate immune system and activated macrophages, thrombus formation and complement activation. In a similar manner, the causes of comorbidities that are emerging as 


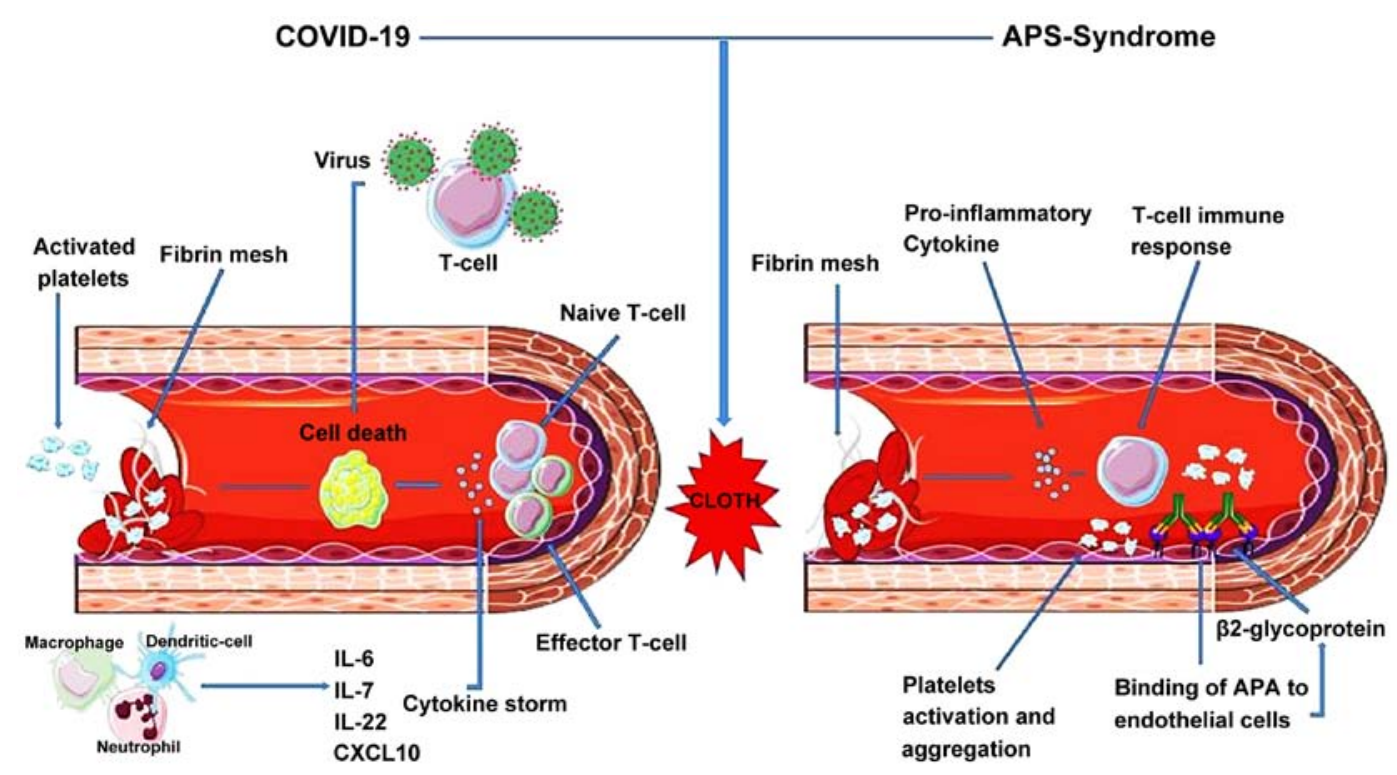

Figure 1. Similarities in the etiopathogenic mechanisms underlying APS and COVID-19. APS, anti-phospholipid antibody syndrome.

capable of worsening the course of COVID-19 infection are remarkably similar to those postulated for the second hit hypothesis of APS and entail immunoinflammatory disorders, such as metabolic syndrome and obesity and hypertension, as well as the use of tobacco. Interestingly, elderly patients have more severe course of COVID-19 infection (9) and exhibit per se higher frequency of aPL-Abs (37).

One would reason that a significant proof substantiating the deductive reasoning would be that defective function of B lymphocytes would ameliorate the course of SARS-CoV-2 infection if the hypothesis that these aPL Abs are involved in the thrombotic diathesis, and this seems actually to be the case, as patients with agammaglobulinemia, who are unable to produce anti-aPL Abs, have a moderate course of the disease (42).

Last, but not least, and lending further support to a deductive exercise of reasoning, both some cases of COVID-19 and APS seem to respond well to anticoagulant treatment. The beneficial effects observed with hydroxychloroquine in APS patients $(26,43)$ has also been claimed in COVID-19 patients but it needs, however, formal demonstration $(43,44)$.

A summary of the similarities between COVID-19 and Thrombosis/APS is presented in Table II and in Fig. 1.

Satisfying Baconian inductivism to substantiate the case. Lending important proof of concept support to the empiric observation that thrombotic phenomena observed during SARS-CoV-2 infection and APS may recognize common pathogenetic pathways, and represent eventually the same nosological entity, is the observation that 3 patients with COVID-19 and ischemic stroke had anti-CL IgA, as well as anti- $\beta 2$-GPI $\operatorname{IgA}$ and $\operatorname{IgG}$ autoantibodies (45). In another article, Beyrouti et al (46) reported 5 cases of ischemic stroke with presence of LA in COVID-19 patients without history of APS. Five of six patients had a positive LA, one with medium-titre IgM aCL and low-titre IgG and IgM anti- $\beta 2$-GPI autoantibodies. Another case report of cerebral stroke with multiple infarctions during COVID-19 infection and associated with aCL Abs has also been reported (47).
These emerging lines of evidence have put forward the hypothesis of performing a routine screening for LA and aPL Abs in patients with COVID-19 infection (48). Along this line of research, another group demonstrated that $45 \%$ of 56 patients infected with COVID-19 were positive for LA, while anti-CL or anti- $\beta 2$-GPI autoantibodies were detected in only 5 out of 50 tested patients (10\%, 3 associated to LA) using IgG and IgM detection (49). However, thrombotic complications were not reported in these patients. It is noteworthy that Zhang et al (45) found IgA autoantibodies directed against $\mathrm{CL}$ or $\beta 2$-GPI, however, it is not clear from the paper whether or not the authors have also searched for the IgA subclass of anti-CL and anti- $\beta 2$-GPI. Finally, other two independent studies have demonstrated that 31 out of 34 (50) and 50 out of 57 patients with COVID-19 infection were positive for LA (51).

Additional important evidence in support of the contribution of secondary APS as potential mechanism of thrombosis during COVID-19 infection was provided by Pineton de Chambrun et al (52) who retrospectively analyzed LA positivity, aCL (IgM, IgG, IgA), anti- $\beta 2$-GPI (IgM, $\mathrm{IgG} / \mathrm{IgA}$ ) and anti-phospholipid (IgM/IgG) autoantibodies in 25 patients with confirmed SARS-CoV-2 infection that were hospitalized at a tertiary ICU in Paris from March 14 to April 8, 2020. The mean age of patients at admission was 47.7 (range, 35-64) and male-to-female ratio was 2.1. All patients had refractory COVID-19-related ARDS requiring extracorporeal membrane oxygenation and receiving non-fractioned heparin with an aimed aPPT ratio of 1.5-2. LA, aCL, anti- $32-\mathrm{GP} 1$ and anti-phospholipid Abs were positive in 23 (92\%), 13 (52\%), 3 (12\%), and 18 (72\%) patients, respectively. The most frequent autoantibody isotype was $\operatorname{IgG}$ for $\mathrm{aCL}(\mathrm{n}=10), \operatorname{Ig} \mathrm{A}$ for anti- $\beta 2$-GPI $(n=3)$ and $\operatorname{IgG}$ for anti-phospholipid $(n=11)$ autoasntibodies. When considering LA positivity with any aCL Abs and any anti- $\beta 2$-GPI single, double and triple positivity was found in $8(32 \%), 13(52 \%)$ and $3(12 \%)$ of the patients. Triple negativity was observed only in 1 patient (4\%). In addition, serum fibrinogen was elevated in most patients $(72 \%)$ at 
DEDUCTION

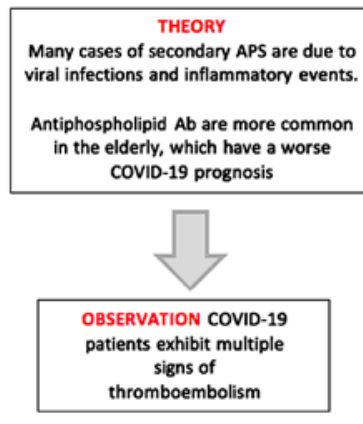

INDUCTION

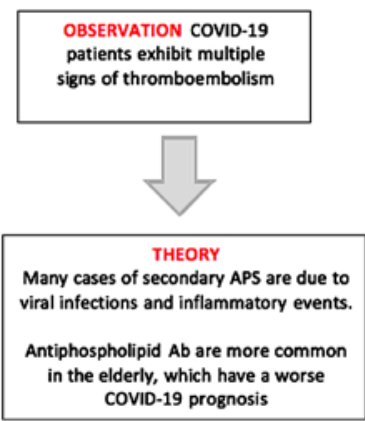

HYPOTHESIS

The thromboembolic diathesis in COVID-19 cases represents secondary form of APS
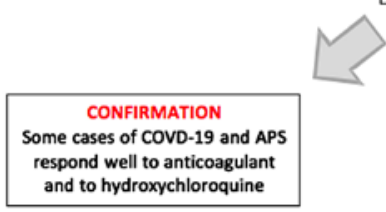

Figure 2. Cartesio deductivism and Baconian inductivism lead to the same hypothesis. APS, anti-phospholipid antibody syndrome.

the time of LA measurement and D-dimer was increased in all the patients. Massive pulmonary embolism was observed in 6 patients that were all aPL Abs positive (52). Considering LA, any anti-cardiolipin and any anti- $\beta 2-\mathrm{GP} 1$ antibodies, 8 (32\%) patients had single APLa positivity, 13 (52\%) had double positivity, $3(12 \%)$ had triple positivity and only one (4\%) was triple negative. Other case reports have also demonstrated that antiCL Abs are associated with thrombosis during COVID-19 infection (53).

However, not all studies have confirmed these initial data. In a study conducted in a Hospital in Madrid in 24 patients with COVID-19 infection and VTE, the Authors found that only two patients $(8.3 \%)$ were weakly positive for aCL $\operatorname{IgM}$ and anti- $\beta 2$-glycoprotein I IgM Abs. Anti-CL IgG and anti- $\beta 2$-glycoprotein I IgG were negative in all patients (54). The reason for these discrepant results are unknown and may be due to different ethnicity and/or different ELISA kits used (54).

Also in light of this latter report, it is clear that several points remain to be studied to fully dismantle the potential contribution of SARS-CoV-2-induced secondary APS to at least some cases of thrombotic events that occur during the infection with this virus. Testing of aPL Abs and LA in a much larger number of COVID-19 patients, as well as the eventual correlation of their titres, with the course of the disease and thrombotic events is mandatory. Potential fluctuation of aPL Abs in response to therapy is of clinical relevance as it may represent an important biomarker. Longitudinal follow-up studies in individuals that have recovered from COVID-19 infection and that are positive for aPL Abs and LA will be important to ascertain whether they revert at recovery of the infection or whether they persist independently. This is particularly so for the elderly patients that are per se at higher risk of thrombosis (55).
Understanding if and to what extent COVID-19 infection induces different production of aPL Abs in the elderly population than in younger individuals is of great relevance as the temporary or persistent presence of these autoantibodies may increase further the thrombotic risk and would require particular therapeutic attention for thromboprophylaxis. Along the same line, routine testing for LA and aPL Abs should be warranted in elderly patients during COVID-19 infection, as these patients are at higher risk of severe infection.

In a similar manner, future studies are required for those individuals with single, dual or triple positivity for LA and aPL Abs that are clinically asymptomatic to prove whether they are at increased risks of thromboembolism in case of COVID-19 infection. Although thromboprophylaxis is currently not approved for these individuals (26) this therapeutic approach may need to be revised during COVID-19 pandemic. The testing of large cohort of COVID-19 patients for aPL Abs is also of outmost relevance to understand the real prevalence of these autoantibodies in patients with SARS-CoV-2. The available data seem to indicate that while the percentage of LA positivity range is approximately $50-80 \%$ of the patients, the positivity for aPL Abs is instead significantly lower and higher for aCL Abs than anti- $\beta 2-$ GPI Abs according to the study of Pineton de Chambrun et al (52).

It has also been suggested that the measurement of aPL Abs in COVID-19 patients may be hindered by the large formation of microparticles (MP) that are released in the circulation. The production of these MPs that are endowed with procoagulant activity is thought to be secondary to the activation induced by cytokines of several cells including platelets, leukocytes, and also of endothelial cells that provokes cell blebbing with the shedding of MPs into the circulation (56).

We strongly believe that the possible definite demonstration that some or most cases of thrombosis triggered by COVID-19 
are secondary forms of APS is not only important for semantic classification or diagnostic criteria but may prove of outmost theranostic relevance for the patients. In particular, on the basis of their positivity for aPL Abs, it would be possible to identify the patients that are at greatest risk for developing thrombotic complications during SARS-CoV-2 infection, including those healthy individuals that are positive for aPL Abs or LA. In addition, understanding whether some fatal and unresponsive cases of DIC observed in COVID-19 may represent cases of CAPS is also of great relevance as considerable experience has been gained during these years in the understanding of immunopathogenesis of CAPS. In particular, though the outcome of CAPS remain unsatisfactory, the generally accepted therapeutic regime consisting of a triple combination entailing anticoagulation, corticosteroids and plasma exchange or intravenous seem to considerably improve the clinical course of patients who received this treatment (40).

A summary of the Deduction and Induction processes that lead to the hypothesis of the occurrence of a secondary form of APS in COVID-19 patients is presented in Fig. 2.

\section{Future therapeutic directions}

Although, the diagnosis of secondary APS would not immediately change the therapeutic approach to prevention and treatment of thrombosis in COVID-19 patients, this demonstration could propel tailored pathogenic approaches for the treatment of a B cell dependent disorders under an unprecedently urgent pandemic situation. Repurposing of specific anti-B cell therapies such as rituximab and ocrelizumab can be anticipated. The anti-C5a mAb eculizumab would also receive attention though its costs would hinder its repurposing in a very large arena such as is that of COVID-19 currently. The use of plasmapheresis should warrant further consideration as well as that of IvIg that seem effective in some cases of APS and CAPS $(26,40)$. Small molecule inhibitors of Toll-like receptor 4 signaling such as resatorvid or GLS-1027 R that may also inhibit neutrophil extracellular traps associated with thrombosis, might be considered in the therapeutic management of COVID-19-related thrombosis $(58,59)$.

Also inhibitors of mTOR pathway such as rapamycin that has been reported to have some beneficial effects in APS and we have identified in silico as a drug candidate for COVID-19 seems of particular interest $(30,60,61)$. Especially so, for the combined ability of rapamycin to exert immunomodulatory and certain antiviral efficacy that remains, however, to be eventually demonstrated on SARS-CoV-2 $(60,62,63)$.

Last, but not least, if some or most cases of COVID-19 associated thrombosis represent a secondary APS, this could give strong impetus to preclinical compounds that represent first class inhibitors of the thrombogenic properties of autoantibodies to $\beta 2-G P 1$. Two of these compounds have demonstrated in vivo efficacy in multiple forms of APS.

The A1-A1 peptide. $\beta 2-\mathrm{GP} 1$ is known to interact with $\mathrm{A} 1$, the first ligand-binding domain of ApoER2. The A1-A1 peptide is a soluble analogue of ApoE receptor 2 with a high affinity for $\beta 2-G P 1 / a n t i b o d y$ complexes. A1-A1 inhibits at least two prothrombotic interactions of $\beta 2-\mathrm{GP} 1 /$ antibody complexes: the binding to ApoER2 and anionic phospholipids on the cellular surfaces. A distinctive feature of A1-A1 compared to $\mathrm{A} 1$ is that A1-A1 preferentially interacts with $\beta 2-\mathrm{GP} 1$ bound to anti- $\beta 2-\mathrm{GP} 1$ antibodies $(64,65)$. Because $\beta 2-\mathrm{GP} 1$ is present in the blood at high concentration, $4 \mu \mathrm{M}$ (66), it is important that a potential drug binds predominantly to pathological

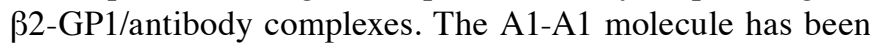
shown to be effective in several rodent models of APS $(64,67,68)$.

AUR-1001. Aur-1001 is a minibody lacking the $\mathrm{CH} 2$ domain and thus incapable of activating the complement that is being developed from Aura Biopharm (Oslo, Norway). The minibody has been shown to have a higher affinity than endogenous anti- $\beta 2-$ GPI autoAbs that are displaced in vitro. AUR-1001 has been shown to be capable of preventing development of both vascular and obstetric APS in preclinical model that are challenged with purified IgG from patients with APS (69).

\section{Acknowledgements}

Not applicable.

\section{Funding}

This study was supported by the current research funds 2020 of IRCCS ‘Centro Neurolesi Bonino-Pulejo' (Messina, Italy).

\section{Availability of data and materials}

Not applicable.

\section{Authors' contributions}

$\mathrm{EC}, \mathrm{PB}, \mathrm{YS}$ and $\mathrm{FN}$ were involved in the conception of the study. AB, RC, AT, AG, CB and PF were involved in the literature search and critical reviewing of the manuscript. EC and FN were involved in the preparation of the draft of the manuscript. $\mathrm{AG}, \mathrm{PF}, \mathrm{CB}, \mathrm{YS}$ and FN were involved in the revising and editing of the manuscript. All authors have read and approved the final manuscript.

\section{Ethics approval and consent to participate}

Not applicable.

\section{Patient consent for publication}

Not applicable.

\section{Competing interests}

FN is cofounder and shareholders of Aura Biopharm. The others authors declare that they have no competing interests.

\section{References}

1. Yuki K, Fujiogi M and Koutsogiannaki S: COVID-19 pathophysiology: A review. Clin Immunol 215: 108427, 2020.

2. Guan WJ, Ni ZY, Hu Y, Liang WH, Ou CQ, He JX, Liu L, Shan H, Lei CL, Hui DSC, et al; China Medical Treatment Expert Group for Covid-19: Clinical characteristics of coronavirus disease 2019 in China. N Engl J Med 382: 1708-1720, 2020. 
3. Rothan HA and Byrareddy SN: The epidemiology and pathogenesis of coronavirus disease (COVID-19) outbreak. J Autoimmun 109: 102433, 2020.

4. Li Y,Zhou W, Yang L and You R: Physiological and pathological regulation of ACE2, the SARS-CoV-2 receptor. Pharmacol Res 157: 104833, 2020.

5. Zhou P, Yang XL, Wang XG, Hu B, Zhang L, Zhang W, Si HR, Zhu Y, Li B, Huang CL, et al: A pneumonia outbreak associated with a new coronavirus of probable bat origin. Nature 579: 270-273, 2020.

6. Doobay MF, Talman LS, Obr TD, Tian X, Davisson RL and Lazartigues E: Differential expression of neuronal ACE2 in transgenic mice with overexpression of the brain renin-angiotensin system. Am J Physiol Regul Integr Comp Physiol 292: R373-R381, 2007.

7. Yan CH, Faraji F, Prajapati DP, Boone CE and DeConde AS: Association of chemosensory dysfunction and COVID-19 in patients presenting with influenza-like symptoms. Int Forum Allergy Rhinol: April 12, 2020 (Epub ahead of print)

8. Li Q, Guan X, Wu P, Wang X, Zhou L, Tong Y, Ren R, Leung KSM, Lau EHY, Wong JY, et al: Early transmission dynamics in Wuhan, China, of novel coronavirus-infected pneumonia. N Engl J Med 382: 1199-1207, 2020.

9. Wang W, Tang J and Wei F: Updated understanding of the outbreak of 2019 novel coronavirus (2019-nCoV) in Wuhan, China. J Med Virol 92: 441-447, 2020.

10. Cascella M, Rajnik M, Cuomo A, Dulebohn SC and Di Napoli R: Features, evaluation and treatment coronavirus (COVID-19). StatPearls Publishing, Treasure Island, FL, 2020.

11. Nishiura H, Jung SM, Linton NM, Kinoshita R, Yang Y, Hayashi K, Kobayashi T, Yuan B and Akhmetzhanov AR: The extent of transmission of novel coronavirus in Wuhan, China, 2020. J Clin Med 9: 330, 2020

12. Tang N, Li D, Wang X and Sun Z: Abnormal coagulation parameters are associated with poor prognosis in patients with novel coronavirus pneumonia. J Thromb Haemost 18: 844-847, 2020.

13. Li LQ, Huang T, Wang YQ, Wang ZP, Liang Y, Huang TB, Zhang HY, Sun W and Wang Y: COVID-19 patients' clinical characteristics, discharge rate, and fatality rate of meta-analysis. J Med Virol 92: 577-583, 2010.

14. Zou Y, Guo H, Zhang Y, Zhang Z, Liu Y, Wang J, Lu H and Qian Z: Analysis of coagulation parameters in patients with COVID-19 in Shanghai, China. Biosci Trends: April 30, 2020 (Epub ahead of print).

15. Lodigiani C, Iapichino G, Carenzo L, Cecconi M, Ferrazzi P, Sebastian T, Kucher N, Studt JD, Sacco C, Alexia B, et al: Venous and arterial thromboembolic complications in COVID-19 patients admitted to an academic hospital in Milan, Italy. Thromb Res 191: 9-14, 2020.

16. Bikdeli B, Madhavan MV, Jimenez D, Chuich T, Dreyfus I, Driggin E, Nigoghossian C, Ageno W, Madjid M, Guo Y, et al; Global COVID-19 Thrombosis Collaborative Group, Endorsed by the ISTH, NATF, ESVM, and the IUA, Supported by the ESC Working Group on Pulmonary Circulation and Right Ventricular Function: COVID-19 and thrombotic or thromboembolic disease: implications for prevention, antithrombotic therapy, and follow-up: JACC State-of-the-Art Review. J Am Coll Cardiol 75: 2950-2973, 2020.

17. Tang N, Bai H, Chen X, Gong J, Li D and Sun Z: Anticoagulant treatment is associated with decreased mortality in severe coronavirus disease 2019 patients with coagulopathy. J Thromb Haemost 18: 18, 2020.

18. Fogarty $\mathrm{H}$, Townsend $\mathrm{L}$, Ni Cheallaigh $\mathrm{C}$, Bergin $\mathrm{C}$, Martin-Loeches I, Browne P, Bacon CL, Gaule R, Gillett A, Byrne M, et al: More on COVID-19 coagulopathy in Caucasian patients. Br J Haematol 189: 1060-1061, 2020.

19. Beun R, Kusadasi N, Sikma M, Westerink J and Huisman A: Thromboembolic events and apparent heparin resistance in patients infected with SARS-CoV-2. Int J Lab Hematol 42 (Suppl 1): 19-20, 2020.

20. Ye Q, Wang B and Mao J: The pathogenesis and treatment of the 'Cytokine Storm' in COVID-19. J Infect 80: 607-613, 2020.

21. Vaninov N: In the eye of the COVID-19 cytokine storm. Nat Rev Immunol 20: 277, 2020

22. Magro C, Mulvey JJ, Berlin D, Nuovo G, Salvatore S, Harp J Baxter-Stoltzfus A and Laurence J: Complement associated microvascular injury and thrombosis in the pathogenesis of severe COVID-19 infection: A report of five cases. Transl Res 220: 1-13, 2020
23. Risitano AM, Mastellos DC, Huber-Lang M, Yancopoulou D, Garlanda C, Ciceri F and Lambris JD: Complement as a target in COVID-19? Nat Rev Immunol 20: 343-344, 2020.

24. Campbell CM and Kahwash R: Will complement inhibition be the new target in treating COVID-19-related systemic thrombosis? Circulation 141: 1739-1741, 2020.

25. Mastaglio S, Ruggeri A, Risitano AM, Angelillo P, Yancopoulou D, Mastellos DC, Huber-Lang M, Piemontese S, Assanelli A, Garlanda C, et al: The first case of COVID-19 treated with the complement C3 inhibitor AMY-101. Clin Immunol 215: 108450, 2020.

26. Rodziewicz M and D'Cruz DP: An update on the management of antiphospholipid syndrome. Ther Adv Musculoskelet Dis 12: 1759720X20910855, 2020.

27. Million M, Bardin N, Bessis S, Nouiakh N, Douliery C, Edouard S, Angelakis E, Bosseray A, Epaulard O, Branger S, et al: Thrombosis and antiphospholipid antibody syndrome during acute Q fever: A cross-sectional study. Medicine (Baltimore) 96: e7578, 2017.

28. Mendoza-Pinto C, García-Carrasco M and Cervera R: Role of infectious diseases in the antiphospholipid syndrome (including its catastrophic variant). Curr Rheumatol Rep 20: 62, 2018.

29. Catoggio C, Alvarez-Uría A, Fernandez PL, Cervera R and Espinosa G: Catastrophic antiphospholipid syndrome triggered by fulminant disseminated herpes simplex infection in a patient with systemic lupus erythematosus. Lupus 21: 1359-1361, 2012.

30. Garcia-Carrasco M, Mendoza-Pinto C, Macias-Diaz S, Vazquez de Lara F, Etchegaray-Morales I, Galvez-Romero JL, Mendez-Martinez S and Cervera R: The role of infectious diseases in the catastrophic antiphospholipid syndrome. Autoimmun Rev 14: 1066-1071, 2015.

31. Gómez-Puerta JA and Cervera R: Diagnosis and classification of the antiphospholipid syndrome. J Autoimmun 48-49: 20-25, 2014.

32. Pengo V, Del Ross T, Ruffatti A, Bison E, Cattini MG, Pontara E, Testa S, Legnani C, Pozzi N, Peterle D, et al: Lupus anticoagulant identifies two distinct groups of patients with different antibody patterns. Thromb Res 172: 172-178, 2018.

33. Cervera R: Antiphospholipid syndrome. Thromb Res 151: (Suppl 1): S43-S47, 2017.

34. Erkan D, Espinosa G and Cervera R: Catastrophic antiphospholipid syndrome: Updated diagnostic algorithms. Autoimmun Rev 10: 74-79, 2010.

35. Shi W, Krilis SA, Chong BH, Gordon S and Chesterman CN: Prevalence of lupus anticoagulant and anticardiolipin antibodies in a healthy population. Aust N Z J Med 20: 231-236, 1990.

36. Fields RA, Toubbeh H, Searles RP and Bankhurst AD: The prevalence of anticardiolipin antibodies in a healthy elderly population and its association with antinuclear antibodies. J Rheumatol 16: 623-625, 1989.

37. Arvanitakis Z,Capuano AW, Brey R, Fleischman DA, Arfanakis K, Buchman AS, Schneider JA, Levine SR and Bennett DA: Antiphospholipid Antibodies: Cognitive and Motor Decline, Neuroimaging and Neuropathology. Neuroepidemiology 53: 100-107, 2019.

38. Ruiz-Irastorza G, Crowther M, Branch W and Khamashta MA: Antiphospholipid syndrome. Lancet 376: 1498-1509, 2010.

39. Carmi O, Berla M, Shoenfeld Y and Levy Y: Diagnosis and management of catastrophic antiphospholipid syndrome. Expert Rev Hematol 10: 365-374, 2017.

40. Rodríguez-Pintó I, Lozano M, Cid J, Espinosa G and Cervera R: Plasma exchange in catastrophic antiphospholipid syndrome. Presse Med 48: 347-353, 2019.

41. Islam MA, Alam F, Wong KK, Kamal MA and Gan SH: Thrombotic management of antiphospholipid syndrome: towards novel targeted therapies. Curr Vasc Pharmacol 15: 313-326, 2017.

42. Quinti I, Lougaris V, Milito C, Cinetto F, Pecoraro A, Mezzaroma I, Mastroianni CM, Turriziani O, Bondioni MP, Filippini M, et al: A possible role for B cells in COVID-19? Lesson from patients with agammaglobulinemia. J Allergy Clin Immunol: April 22, 2020 (Epub ahead of print).

43. McKee DL, Sternberg A, Stange U, Laufer S and Naujokat C: Candidate drugs against SARS-CoV-2 and COVID-19. Pharmacol Res 157: 104859, 2020.

44. Chighizola CB, Andreoli L, Gerosa M, Tincani A, Ruffatti A and Meroni PL: The treatment of anti-phospholipid syndrome: a comprehensive clinical approach. J Autoimmun 90: 1-27, 2018.

45. Zhang Y, Xiao M, Zhang S, Xia P, Cao W, Jiang W, Chen H, Ding X, Zhao H, Zhang H, et al: Coagulopathy and antiphospholipid antibodies in patients with Covid-19. N Engl J Med 382: e38, 2020. 
46. Beyrouti R, Adams ME, Benjamin L, Cohen H, Farmer SF, Goh YY, Humphries F, Jäger HR, Losseff NA, Perry RJ, et al: Characteristics of ischaemic stroke associated with COVID-19. Neurol Neurosurg Psychiatry: April 30,2020 (Epub ahead of print). 47. Zayet S, Klopfenstein T, Kovacs R, Stancescu S and Hagenkötter B: Acute cerebral stroke with multiple infarctions and COVID-19, France, 2020. Emerg Infect Dis 26: 26, 2020.

48. Aubignat M and Godefroy O: COVID-19 and ischemic stroke: Should we systematically look for lupus anticoagulant and antiphospholipid antibodies? Rev Neurol (Paris) 176: 505-506, 2020.

49. Harzallah I, Debliquis A and Drénou B: Lupus anticoagulant is frequent in patients with Covid-19. J Thromb Haemost, 2020

50. Bowles L, Platton S, Yartey N, Dave M, Lee K, Hart DP, MacDonald V, Green L, Sivapalaratnam S, Pasi KJ, et al: Lupus anticoagulant and abnormal coagulation tests in patients with Covid-19. N Engl J Med: May 5, 2020 (Epub ahead of print).

51. Helms J, Tacquard C, Severac F, Leonard-Lorant I, Ohana M, Delabranche X, Merdji H, Clere-Jehl R, Schenck M, Fagot Gandet F, et al; CRICS TRIGGERSEP Group (Clinical Research in Intensive Care and Sepsis Trial Group for Global Evaluation and Research in Sepsis): High risk of thrombosis in patients with severe SARS-CoV-2 infection: A multicenter prospective cohort study. Intensive Care Med 46: 1089-1098, 2020.

52. Pineton de Chambrun M, Frere C, Miyara M, Amoura Z, Martin-Toutain I, Mathian A, Hekimian G and Combes A: High frequency of antiphospholipid antibodies in critically-ill COVID-19 patients: a link with hypercoagulability? J Intern Med: June 12, 2020 (Epub ahead of print).

53. Hossri S, Shadi M, Hamarsha Z, Schneider R and El-Sayegh D: Clinically significant anticardiolipin antibodies associated with COVID-19. J Crit Care 59: 32-34, 2020.

54. Galeano-Valle F, Oblitas CM, Ferreiro-Mazón MM, Alonso-Muñoz J, Del Toro-Cervera J, di Natale M and Demelo-Rodríguez P: Antiphospholipid antibodies are not elevated in patients with severe COVID-19 pneumonia and venous thromboembolism. Thromb Res 192: 113-115, 2020.

55. Chaudhary R, Pagali S, Garg J, Murad MH, Wysokinski WE and McBane RD 2nd: DOACs versus VKAs in older adults treated for acute venous thromboembolism: systematic review and metaanalysis. J Am Geriatr Soc: May 22, 2020 (Epub ahead of print)

56. Marchandot B, Sattler L, Jesel L, Matsushita K, Schini-Kerth V, Grunebaum L and Morel O: COVID-19 Related coagulopathy: A distinct entity? J Clin Med 9: 9, 2020.

57. Oxley TJ, Mocco J, Majidi S, Kellner CP, Shoirah H, Singh IP, De Leacy RA, Shigematsu T, Ladner TR, Yaeger KA, et al: Large-vessel stroke as a presenting feature of Covid-19 in the young. N Engl J Med 382: e60, 2020.

58. Plunk MA, Alaniz A, Olademehin OP, Ellington TL, Shuford KL and Kane RR: Design and Catalyzed Activation of Tak-242 Prodrugs for Localized Inhibition of TLR4-Induced Inflammation. ACS Med Chem Lett 11: 141-146, 2020.

59. Stojanovic I, Cuzzocrea S, Mangano K, Mazzon E, Miljkovic D, Wang M, Donia M, Al Abed Y, Kim J, Nicoletti F, et al: In vitro, ex vivo and in vivo immunopharmacological activities of the isoxazoline compound VGX-1027: Modulation of cytokine synthesis and prevention of both organ-specific and systemic autoimmune diseases in murine models. Clin Immunol 123 : $311-323,2007$
60. Nicoletti F, Fagone P, Meroni P, McCubrey J and Bendtzen K: mTOR as a multifunctional therapeutic target in HIV infection. Drug Discov Today 16: 715-721, 2011.

61. Fagone P, Ciurleo R, Lombardo SD, Iacobello C, Palermo CI, Shoenfeld $Y$, Bendtzen $K$, Bramanti $P$ and Nicoletti F. Transcriptional landscape of SARS-CoV-2 infection dismantles pathogenic pathways activated by the virus, proposes unique sex-specific differences and predicts tailored therapeutic strategies. Autoimmun Rev 19: 102571, 2020.

62. Nicoletti F, Lapenta C, Donati S, Spada M, Ranazzi A, Cacopardo B, Mangano K, Belardelli F, Perno C and Aquaro S: Inhibition of human immunodeficiency virus (HIV-1) infection in human peripheral blood leucocytes-SCID reconstituted mice by rapamycin. Clin Exp Immunol 155: 28-34, 2009.

63. Maiese K: The mechanistic target of rapamycin (mTOR): Novel considerations as an antiviral treatment and possibilities for COVID-19. Curr Neurovasc Res: April 25, 2020 (Epub ahead of print).

64. Kolyada A, Lee CJ, De Biasio A and Beglova N: A novel dimeric inhibitor targeting Beta2GPI in Beta2GPI/antibody complexes implicated in antiphospholipid syndrome. PLoS One 5: e15345, 2010.

65. Lee CJ, De Biasio A and Beglova N: Mode of interaction between $\beta 2$ GPI and lipoprotein receptors suggests mutually exclusive binding of $\beta 2 \mathrm{GPI}$ to the receptors and anionic phospholipids. Structure 18: 366-376, 2010.

66. Lin F, Murphy R, White B, Kelly J, Feighery C, Doyle R, Pittock S, Moroney J, Smith O, Livingstone W, et al: Circulating levels of $\beta 2$-glycoprotein I in thrombotic disorders and in inflammation. Lupus 15: 87-93, 2006.

67. Kolyada A, Karageorgos I, Mahlawat P and Beglova N: An A1-A1 mutant with improved binding and inhibition of $\beta 2 \mathrm{GPI} / \mathrm{antibody}$ complexes in antiphospholipid syndrome. FEBS J 282: 864-873, 2015.

68. Kolyada A, Porter A and Beglova N: Inhibition of thrombotic properties of persistent autoimmune anti- $\beta 2 \mathrm{GPI}$ antibodies in the mouse model of antiphospholipid syndrome. Blood 123: 1090-1097, 2014

69. Agostinis C, Durigutto P, Sblattero D, Borghi MO, Grossi C, Guida F, Bulla R, Macor P, Pregnolato F, Meroni PL, et al: A non-complement-fixing antibody to $\beta 2$ glycoprotein I as a novel therapy for antiphospholipid syndrome. Blood 123: 3478-3487, 2014.

This work is licensed under a Creative Commons Attribution 4.0 International (CC BY 4.0) License. 